\title{
Slot-Die-Printed Sodium-Embedded Alumina Enables High-Performance, Wafer-Scale MoS2 Electronics
}

\section{Yonghyun Kwon}

Yonsei University

Jihyun Kim

Sungkyunkwan University

\section{Sae Byeok Jo}

Sungkyunkwan University https://orcid.org/0000-0001-7383-3546

Dong Gue Roe

Yonsei University

\section{Dongjoon Rhee}

Sungkyunkwan University

\section{Dae Woo Kim}

Yonsei University

\section{Joohoon Kang}

Sungkyunkwan University (SKKU) https://orcid.org/0000-0002-6578-2547

Jeong Ho Cho ( $\sim$ jhcho94@yonsei.ac.kr)

Yonsei University https://orcid.org/0000-0002-1030-9920

\section{Article}

Keywords:

Posted Date: February 1st, 2022

DOI: https://doi.org/10.21203/rs.3.rs-1048728/v1

License: (1) (1) This work is licensed under a Creative Commons Attribution 4.0 International License. Read Full License 


\section{Abstract}

Two-dimensional nanomaterials offer a promising new avenue in the forthcoming era of Beyond Moore electronics, due to their unique feasibility for forming van der Waals contacts alleviating detrimental interfacial electronics losses. However, their scalable production via solution processing compels the competition between achieved material quality and scalability, leaving them incompetent for practical electronics applications. Here, we, for the first time, have realized a large-scale solution-processed $\mathrm{MoS}_{2}$ transistor arrays via slot-die printing, in which devices exhibited exceptionally high average electronic mobility exceeding $100 \mathrm{~cm}^{2} \mathrm{~V}^{-1} \mathrm{~s}^{-1}$. A set of comprehensive temperature-dependent and spatialdistribution characteristics analyses revealed that the use of sodium-embedded alumina (SEA) as the gate dielectric enabled the charge transport on $\mathrm{MoS}_{2}$ through long-range charge percolations among low density deep charged-traps, allowing transistor performance to be dramatically boosted. Furthermore, we have successfully demonstrated various functional logic gates, NOT, NOR, NAND and SRAM, via waferscale homogeneous slot-die printing of both $\mathrm{MoS}_{2}$ and SEA.

\section{Background}

Solution-based processing of low-dimensional materials has been considered as a promising route toward scalable synthesis for their use in practical electronics in a cost-effective manner ${ }^{1-5}$. In general, desired nanomaterials are stabilized in a solvent as an ink form via wet chemistry ${ }^{6-8}$, and subsequently deposited on a target substrate using readily available coating techniques (e.g., spin coating, spray coating, vacuum filtration, and inkjet printing) ${ }^{9,10}$. Among various candidates, two-dimensional (2D) nanosheets have been considered as promising solution-processable materials for use in large-scale electronics ${ }^{11-13}$. In particular, the possibility of having atomically clean, dangling bonds-free surfaces renders them uniquely feasible for forming van der Waals (vdW) contacts with neighboring nanosheets, which enables minimized charge transport loss at the interfaces when they form thin-film network structures ${ }^{14,15}$. Furthermore, in the similar manner to OD quantum dots and 1D CNTs, a wide range of 2D nanomaterials possessing various electronic properties can be exfoliated from their bulk crystals and dispersed in a solvent as a stable ink. ${ }^{16,17}$

Among the 2D family, semiconducting molybdenum disulfide $\left(\mathrm{MoS}_{2}\right)$ has been most intensively studied as a potential candidate for next-generation electronics due to their intriguing physical, chemical, and optical properties ${ }^{18-20}$. However, $\mathrm{MoS}_{2}$ has not been fully implemented in practical demonstrations yet due to difficulties in minimizing the trade-off between scalable synthesis and material quality. For example, the liquid-phase exfoliation enabled a large-scale production of dispersed $\mathrm{MoS}_{2}$ nanosheets, but the small lateral size $(<100 \mathrm{~nm})$ of exfoliated nanosheets hindered effective formation of an electrically conductive thin-film structure. Instead, molecular intercalation-based electrochemical exfoliation method offered an alternative scalable route for the homogeneous production of relatively large $(>1 \mu \mathrm{m})$ fewlayered $\mathrm{MoS}_{2}$ nanosheets, but the field-effect mobility of their wafer-scale transistor arrays has been 
limited to $10 \mathrm{~cm}^{2} \mathrm{~V}^{-1} \mathrm{~s}^{-1}$ at room temperature ${ }^{13}$. Although this value was comparable to those of micromechanically-exfoliated $\mathrm{MoS}_{2}$ devices fabricated on conventional oxidized silicon substrates, it still lags far behind the phonon-scattering-limited field-effect mobility of bulk $\mathrm{MoS}_{2}$ placed in the range of $200-500 \mathrm{~cm}^{2} \mathrm{~V}^{-1} \mathrm{~s}^{-1}$ at room temperature ${ }^{21}$. One possible solution to maximize the field-effect mobility of solution-processed $\mathrm{MoS}_{2}$ thin-film is employing high-k dielectric layers such as $\mathrm{HfO}_{2}$ and $\mathrm{Al}_{2} \mathrm{O}_{3}{ }^{22-25}$. In the previous report, for example, the average field-effect mobility of micromechanically exfoliated $\mathrm{MoS}_{2}$ nanosheets has been significantly improved more than two orders of magnitude by introducing $\mathrm{HfO}_{2}$ grown by atomic layer deposition (ALD) as a high- $k$ gate dielectric ${ }^{11}$. Although ALD can produce dielectric layers over large areas in high quality, the process is limited by slow deposition rate and difficulties achieving controlled doping because the reactions between the precursors are complicated. ${ }^{26,27}$

In this work, we demonstrate a wafer-scale solution-processed $\mathrm{MoS}_{2}$ thin-film transistor array with average field-effect electron mobility exceeding $100 \mathrm{~cm}^{2} \mathrm{~V}^{-1} \mathrm{~s}^{-1}$ at room temperature. The consecutive slot-die printings of sodium-embedded alumina (SEA) and $\mathrm{MoS}_{2}$ nanosheet inks were employed for the scalable construction of gate dielectric and semiconducting layers on wafer, respectively. A comprehensive suite of analyses based on temperature-dependent charge transport behavior and scanning probe microscopy revealed that the exceptionally high electron mobility of $\mathrm{MoS}_{2}$ on SEA was attributed to the low-density incursion of deep charged impurities with reduced shallow electronic fluctuations, which allowed the predominance of long-range percolation of charges. Finally, as a demonstration of scalable thin-film formation with extremely low operational cost at room temperature, various functional logic gates including NOT, NOR, NAND, and SRAM are presented in wafer scale.

\section{Device Fabrication Based On Slot-die Coating}

To realize wafer-scale fabrication of $\mathrm{MoS}_{2}$-based field-effect transistor (FET) arrays, both sodiumembedded alumina (SEA) precursor solution and $\mathrm{MoS}_{2}$ flake dispersion are sequentially coated on an arbitrary rigid substrate via a slot-die coating method as illustrated in Figure 1a (photographs of asprepared dispersions, $\mathrm{MoS}_{2}$ flake morphology, and films coated on transparent glass substrates are shown in Supplementary Figs. 1 and 2). An optical image of the slot-die coater for preparing wafer-scale electronics is shown in Figure 1b. First, SEA precursor solution was carefully injected into the head at a constant rate of $2.5 \mathrm{~mL} \mathrm{~min}{ }^{-1}$ and ejected through the nozzle placed at the bottom of the head. While the solution is ejecting, the slot-die head is simultaneously moving in $x-y$ directions at a constant rate of $\sim 10$ $\mathrm{mm} \mathrm{s}^{-1}$ for homogeneous coating of SEA on the substrate. Subsequently, MoS 2 dispersion was coated in the similar manner to form a semiconducting layer on the SEA layer (Supplementary Video 1). For successful formation of the semiconducting layer, $\mathrm{MoS}_{2}$ dispersion was prepared by molecular intercalation-driven electrochemical exfoliation followed by a mild bath sonication in a solvent. Photographs taken after each layer coating on a 5-inch Si wafer are shown in Figure 1c. The resulting $\mathrm{MoS}_{2}$ /SEA layers were subjected to fabricate a conventional FET structure as illustrated in Figure 1d, where the corresponding atomic structures of $\mathrm{MoS}_{2}$ flake and SEA are represented. Based on the 
structure, a representative electrical transfer curve on a semi-logarithmic scale (red curves) and a linear scale (blue curve) is shown in Figure 1e. As the gate voltage sweeps to the positive values, the drain current turns on abruptly at $\mathrm{V}_{\mathrm{G}} \approx 0 \mathrm{~V}$ with a high on/off ratio $\left(>10^{5}\right)$ and a negligible hysteresis loop during the forward and reverse sweeps. The corresponding output curve exhibit a typical n-type FET behavior having the drain current increase and eventually saturate as the drain voltage increases (Supplementary Fig. 3). From the transfer characteristics, field-effect carrier mobility values are extracted based on the equation,

$$
I_{D}=C_{i} \mu W\left(V_{G}-V_{t h}\right) / L
$$

where $I_{D}$ is the drain current; $C_{i}$ is the effective capacitance of gate insulator; $V_{\mathrm{G}}$ and $V_{\mathrm{th}}$ are the gate voltage and threshold voltage, respectively; $L$ and $W$ are the channel length and width, respectively. The carrier mobilities of 49 transistors in a fabricated array are shown in Figure $1 \mathbf{f}$ as a two-dimensional (2D) color mapping, which shows high spatial homogeneity with an average value of $\sim 112 \pm 4.6 \mathrm{~cm}^{2} \mathrm{~V}^{-1} \mathrm{~s}^{-1}$ at room temperature (the transfer curves for all 49 FETs are shown in Supplementary Fig. 4). This is the record-high mobility among wafer-scale $\mathrm{MoS}_{2}$-based FETs reported to date, and the processing temperature for $\mathrm{MoS}_{2}$ channel formation $\left(250^{\circ} \mathrm{C}\right)$ is much lower compared to CVD-based devices (Supplementary Fig. 5 and Supplementary Table 1). Color maps and statistics of carrier mobility, turn-on voltage, and current on/off ratio are shown in Supplementary Figs. 6 and 7. To elucidate the origin of high carrier mobilities, effective capacitance values of the slot-die coated SEA dielectric layer were measured by forming a metal-insulator-metal (MIM) structure with thermally deposited $\mathrm{Cr} / \mathrm{Au}$ electrodes placed on top. The resulting frequency-dependent capacitance and phase angle measured by an impedance spectroscopy are shown in Figure 1g. Notably, the specific capacitance measured at $1 \mathrm{~Hz}$ is $\sim 0.9 \mu \mathrm{F} \mathrm{cm}^{-2}$, which is 18 times higher than that of an $\mathrm{Al}_{2} \mathrm{O}_{3}$ dielectric with a comparable thickness $\left.(\sim 0.05 \mu \mathrm{F} \mathrm{cm})^{-2}\right)^{28}$. To understand the origin of the high capacitance, we explored frequency dependence. The capacitance is nearly constant until the frequency increases up to $100 \mathrm{~Hz}$, and then gradually decreases as the frequency further increases. For the same frequency range, the measured phase angle also goes toward $0^{\circ}$ and gradually returns to $-90^{\circ}$ when the frequency increases, which implies that the dominant working mechanism changes from capacitive $\left(-90^{\circ}\right)$ to resistive (toward $0^{\circ}$ ) and back to capacitive operation. ${ }^{29-32}$ These frequency-dependent capacitance and phase angle changes are mainly attributed to the displacement of $\mathrm{Na}^{+}$ions embedded in alumina and their limited polarization response time. ${ }^{33-36}$ Eventually, the measured capacitance values become close to $\sim 0.1 \mu \mathrm{F} \mathrm{cm}^{-2}$, which is similar to the reported capacitance of the pristine $\mathrm{Al}_{2} \mathrm{O}_{3}$. Figure $1 \mathrm{~h}$ shows the distribution of 49 capacitance measurements, where the color intensity indicates the value of measured capacitance and $x$ and $y$ axis designate spatial positions. Based on the spatial mapping, an average capacitance value of $0.907 \pm$ $0.002 \mu \mathrm{F} \mathrm{cm}^{-2}$ was shown with high spatial uniformity $\left(0.055 \mu \mathrm{F} \mathrm{cm}{ }^{-2}\right)$.

Based on the SEA layer possessing a high average capacitance, we further explored solution-processed $\mathrm{MoS}_{2}$-based electronics to investigate the origin of the working principle and maximize the device performance. As we illustrated in Figure 1, SEA and $\mathrm{MoS}_{2}$ layers are sequentially coated via slot-die 
coating, and subsequently the $\mathrm{MoS}_{2}$ layer is subjected for chemical doping by exposure to bis(trifluoromethane) sulfonimide (TFSI) followed by heat treatment (Figure 2a). The resulting electrical transfer characteristics are shown in Figure $\mathbf{2} \mathbf{b}$ after each step (i.e., MoS $_{2}$ coating, TFSI treatment, and heat treatment). First, the $\mathrm{MoS}_{2}$ ink concentrations are varied from $1 \mathrm{mg} \mathrm{mL}^{-1}$ to $3 \mathrm{mg} \mathrm{mL}^{-}$ ${ }^{1}$ to optimize the slot-die-coated channel thickness (Supplementary Fig. 8). Based on the electrical characteristics of each thickness, we decided to use $2 \mathrm{mg} \mathrm{mL}^{-1}$ for the rest of the device fabrication (Supplementary Fig. 9). While the as-coated $\mathrm{MoS}_{2}$ thin-film shows a current on/off ratio of $\sim 7 \times 10^{2}$, subthreshold swing (SS) of $1.58 \mathrm{~V} \mathrm{dec}^{-1}$, and threshold voltage $\left(\mathrm{V}_{\mathrm{th}}\right)$ of $0.16 \mathrm{~V}$ with a heavily $\mathrm{n}$-doped electrical behavior due to the sulfur vacancies formed during the processing (gray line), the unintentional doping is significantly recovered by TFSI-assisted chemical treatment (black line). After the chemical treatment, the transfer characteristics significantly changed with the current on/off ratio of $\sim 10^{5}$, SS of $0.32 \mathrm{~V} \mathrm{dec}^{-1}$, and positively shifted $\mathrm{V}_{\text {th }}$ of $3.23 \mathrm{~V}$. As previously reported, TFSI molecules play a role to passivate sulfur vacancies on the basal plane of $\mathrm{MoS}_{2}$ nanosheets, which act as carrier trapping sites ${ }^{13}$. Also, the charge density of $\mathrm{MoS}_{2}$ is reduced because the TFSI molecules serve as a Lewis acid to withdraw electrons ${ }^{37}$. Therefore, as the resulting transfer characteristic curve shows, the TFSI-treated $M_{0} S_{2}$ device shows SS improved $\sim 5$ times and $V_{\text {th }}$ shifted toward a positive direction compared to those of as-coated $\mathrm{MoS}_{2}$ thin-film counterpart. The electrical characteristics corresponding to the TFSI treatments with various concentrations are shown in Supplementary Fig. 10. A comprehensive suite of characterizations including Kelvin probe force microscopy (KPFM), Raman spectroscopy, Photoluminescence (PL) spectroscopy and $x$-ray photoelectron spectroscopy (XPS) is also performed to reveal the effect of TFSI concentration on the properties of the $\mathrm{MoS}_{2}$ thin-film (Supplementary Fig. 1113). Following the chemical treatment, an additional annealing process further improves $\mathrm{MoS}_{2}$ nanosheet-to-nanosheet contact and removes residual solvent, and then the resulting current level improves at least one order of magnitude at the applied gate voltage of $5 \mathrm{~V}$ (red line). Transfer characteristics at various annealing temperatures $\left(100{ }^{\circ} \mathrm{C}, 150{ }^{\circ} \mathrm{C}, 200{ }^{\circ} \mathrm{C}, 250{ }^{\circ} \mathrm{C}\right.$, and $\left.300{ }^{\circ} \mathrm{C}\right)$ are plotted in Supplementary Fig. 14a. As the annealing temperature increases up to $250^{\circ} \mathrm{C}$, on current increases and $V_{\text {th }}$ shifts to negative, and then the device performance is slightly degraded after annealing at $300{ }^{\circ} \mathrm{C}$. The corresponding carrier mobility and current on/off ratio as a function of annealing temperature are plotted in Supplementary Fig. 14b, and the corresponding work function distribution are plotted in Supplementary Fig. 15. The decreased carrier mobility and increased off current of the device annealed at $300{ }^{\circ} \mathrm{C}$ are possibly due to the partial oxidation of $\mathrm{MoS}_{2}$ to $\mathrm{MoO}_{\mathrm{x}}{ }^{38}$.

To elucidate the origin of the ultrahigh carrier mobility $\left(>100 \mathrm{~cm}^{2} \mathrm{~V}^{-1} \mathrm{~s}^{-1}\right)$ of our solution-processed $\mathrm{MoS}_{2}{ }^{-}$ based transistors, thorough analysis has been performed on the SEA dielectrics along with alumina $\left(\mathrm{Al}_{2} \mathrm{O}_{3}\right)$ dielectrics without sodium, which we used as control samples for comparison in depth. The alumina dielectric layers were prepared in two conventional ways: atomic layer deposition (ALD) and solution processing (spin-coated). $\mathrm{MoS}_{2}$ FETs fabricated on both alumina dielectrics show an average carrier mobility of $\sim 10 \mathrm{~cm}^{2} \mathrm{~V}^{-1} \mathrm{~s}^{-1}$, which is comparable to the previously reported value ${ }^{13}$. To reveal 
charge transport mechanisms, we further investigated electrical characteristics of the devices as a function of temperature. Frequency-dependent dielectric properties of the SEA at different temperatures are shown in Supplementary Fig. 16. Transfer curves of $\mathrm{MoS}_{2}$ FETs based on SEA and control alumina dielectrics were also measured as a function of temperature (Supplementary Figs. 17 and 18). Figure 2c shows temperature-dependent electrical conductivity ( $\sigma$ ) of $\mathrm{MoS}_{2}$ FETs fabricated on the SEA dielectric layer at different gate voltages extracted from the temperature-dependent transfer curves (Supplementary Fig. 17). The resulting curves are well-fitted with the 2D variable range hopping (2D VRH) model below $280 \mathrm{~K}$ with an equation $\sigma \sim \exp \left[-\left(\frac{T_{1}}{T}\right)^{\frac{1}{3}}\right]$; temperature, $T_{1}$ is a constant related to the size of $2 \mathrm{D}$ localized states $\left(a, T_{1} \sim a^{2}\right) .{ }^{39,40}$ In Supplementary Fig. 19, both control samples fabricated on solution-processed and ALD alumina dielectrics are also fitted using the 2D VRH model extracted from the temperature-dependent transfer curves (Supplementary Fig.

18). Figure $2 \mathbf{d}$ shows the equivalent carrier concentrations extracted from an equation

$$
n=C_{i}\left(V_{G}-V_{t h}\right)
$$
and the corresponding $T_{1}$ values. SEA-gated MoS 2 FETs show higher $T_{1}$ values compared to aluminagated counterparts, which implies wider localization length for the case with SEA ${ }^{41}$. At high temperatures, electrical conductance of the $\mathrm{Al}_{2} \mathrm{O}_{3}$-gated $\mathrm{MoS}_{2}$ FETs show stronger temperature dependence, which is possibly attributed to the transition from variable-range hopping to nearest-neighbor hopping $(\mathrm{NNH})$ mechanism 40,42 . The NNH can be fitted by the Arrhenius equation $\sigma \sim \exp \left[-\left(\frac{T_{0}}{T}\right)\right]$, where $\sigma$ is the conductivity of semiconductor channel, $T$ is the temperature, $T_{0}$ represents the activation energy for carrier. The $T_{0}$ values extracted from the equation are plotted in Figure $2 \mathrm{e}$. Higher $T_{0}$ values of $\mathrm{MoS}_{2}$ FETs on the SEA layer indicate that deep trap states exist in the SEA. These results suggest that the charge transport in $\mathrm{MoS}_{2}$ on SEA is largely governed by the long-range percolation through deep traps, assuming the constant density of states near the Fermi edge. Moreover, considering that the ratio of characteristic temperatures $\left(\gamma=T_{1} / T_{0}\right)$ reflects the ratio between intersite distance $\left(d_{\mathrm{NN}}\right)$ and $a$, higher $\gamma$ along with higher $a$ indicates that the density of traps in SEA-gated $\mathrm{MoS}_{2}$ devices is lower compared to the alumina-gated $\mathrm{MoS}_{2}$ cases (Supplementary Fig. 20). In this sense, we further clarified the origin of 2D VRH behavior based on the KPFM analysis ${ }^{43}$.

The top image of Figure $\mathbf{2} \mathbf{f}$ shows the work function distribution of SEA surface measured using KPFM. Slightly bright and dark spots were observed throughout the whole plane, while no such distribution was observed on $\mathrm{Al}_{2} \mathrm{O}_{3}$ (Supplementary Fig. 21a). To obtain more detailed spatial distribution of work functions on our dielectric film, we performed 2D fast Fourier transform (2D FFT) of the measured work function distribution (Figure 2f, bottom image). The yellow/green spots visible on the 2D FFT plot imply that regions with more than $10 \mathrm{meV}$ difference in work functions compared to the surrounding areas are periodically appearing. In the case of SEA, the yellow/green spots in the 2D FFT images are observed at 
frequencies close to $0.1 \mathrm{~nm}^{-1}$. For $\mathrm{Al}_{2} \mathrm{O}_{3}$ (Supplementary Fig. 21b), the yellow/green spots are highly concentrated at frequencies close to $0.8 \mathrm{~nm}^{-1}$. The distribution of work function was fitted by gaussian plot and shown in Figure $\mathbf{2 g}$, which showed a slight broadening in the distribution of work function in SEA dielectric compared to $\mathrm{Al}_{2} \mathrm{O}_{3}$. On the other hand, the distribution of amplitudes from the 2D FFT images (Figure 2h) clearly demonstrates larger densities in $\mathrm{Al}_{2} \mathrm{O}_{3}$ than SEA for the local fluctuations with low amplitudes $\left(<10^{-3} \mathrm{eV}\right)$. At higher amplitudes $\left(>10^{-3} \mathrm{eV}\right)$, SEA showed larger densities. It can be reasonably deduced that $\mathrm{Al}_{2} \mathrm{O}_{3}$ has a strong oscillating work function distribution with high frequencies but small amplitudes, and SEA shows a weak oscillating work function distribution with low frequencies but large amplitudes. Also, oscillating work function distribution with small amplitudes exists much less in SEA (Figure 2h). By summing up the information, we can roughly estimate the density of state (DOS) as well as their spatial distribution for $\mathrm{Al}_{2} \mathrm{O}_{3}$-gated and SEA-gated $\mathrm{MoS}_{2}$ devices, as schematically illustrated in

Figure 2i. The low-density incursion of deep trap states along with reduced shallow electronic fluctuations explains the large temperature dependence of SEA-gated $\mathrm{MoS}_{2}$ transistors as well as the dominance of long-range percolations, which agrees with the highly boosted charge carrier mobility achieved in our system.

\section{Applicability To Large-area Logic Circuits}

In order to verify the applicability of our transistors to large area electronics, various logic circuits were fabricated and tested: NOT, NOR, NAND, and SRAM (Figure 3a). The circuit diagram of a NOT gate (inverter) is illustrated in Figure 3b. Two identical $\mathrm{MoS}_{2}$ transistors were used as both driver and load transistor. Voltage transfer characteristics at different supply voltages $\left(V_{\mathrm{DD}}\right)$ and the resulting signal gain are shown in Figure $3 c$. An output voltage equal to the supply voltage was measured at low input voltages (close to $0 \mathrm{~V}$ ), which corresponds to the logic state " 1 ". The output voltage decreased to $0 \mathrm{~V}$ as the input voltage increased, which corresponds to the logic state " 0 ". This clear inversion of voltage confirms good NOT gate operation. Signal gain (absolute value of $\mathrm{d} V_{\text {OUT }} / \mathrm{d} V_{\mathbb{I N}}$ ) higher than 7 was achieved. Figure 3d shows circuit diagrams of NAND and NOR gates. Both NOR and NAND gates utilize three identical $\mathrm{MoS}_{2}$ transistors; two in parallel and one in series for NOR gate, all three in series for NAND gate. In a NAND gate, the output voltage is $2 \mathrm{~V}$ (logic sate "1") when at least one of the input voltages are $0 \mathrm{~V}$ (logic state " 0 "). And the output is at logic state " 0 " only when both of the inputs are at logic state " 1 ". For a NOR gate, the output is at logic state " 1 " only when both inputs are at logic state " 0 ". For the rest of the possible input logic combinations (i.e. $(1,0),(0,1),(1,1))$, output logic state is at " 0 ". Output voltages for both NAND (top) and NOR (bottom) gates are plotted in Figure 3e. Both NAND and NOR gates steadily exhibited correct output logic states for all possible input logic combinations over few repetitions. SRAM is composed of two cross connected inverters, which contain two transistors each (Figure $\mathbf{3 f}$ ). In an SRAM cell, each output of an inverter is connected to the input of the other. When an initial input voltage (low or high) is applied to an inverter, the resulting output voltage (logic state 1 or 0 ) is fed to the input of the cross connected inverter. Then, the output logic state of the cross connected inverter is equal to the initially applied input, which is looped back into the initially operated inverter. Thus, a SRAM cell can 
maintain its output level even after the input is completely opened. Figure $\mathbf{3 g}$ display the operation of SRAM at various input voltages and time span, where the shaded regions represent completely open input states. Complete maintenance of output states at open input states (shaded regions) confirms excellent operation of our fabricated SRAM cell.

\section{Conclusion}

To summarize, we have realized large-scale, solution-processed and high performance $\mathrm{MoS}_{2}$ electronics on a 5-inch wafer by employing consecutive slot-die printings of $\mathrm{MoS}_{2}$ nanosheet and SEA inks for semiconducting and insulating layers, respectively. The solution-processed $\mathrm{MoS}_{2}$ FETs on the SEA layer exhibited an exceptionally high field-effect mobility exceeding $100 \mathrm{~cm}^{2} \mathrm{~V}^{-1} \mathrm{~s}^{-1}$, which is more than 10 times higher than the previously reported values ${ }^{13}$. The origin of such high carrier mobility was thoroughly elucidated by a comprehensive suite of analysis with respect to temperature dependence of electronic characteristics as well as spatial distribution of surface characteristics. Furthermore, the high spatial uniformity of the device performance successfully enabled to demonstrate logic circuits including NOT, NAND, NOR, and SRAM. Thus, this work can provide a new pathway for high-performance, large-area electronics based on solution-processed low dimensional materials.

\section{Methods}

\section{Device fabrication}

To prepare sodium-embedded alumina (SEA) precursor solutions, aluminum nitrate nonahydrate ( $98 \%$, Sigma-Aldrich) and sodium hydrogen sulfate (Sigma-Aldrich) mixed at a molar ratio of 10:2 was dissolved in a binary solvent of ethanol (anhydrous, Sigma-Aldrich) and acetone (99.5\%, Sigma-Aldrich) (volume ratio $=6: 1$ ). The final concentration was $0.6 \mathrm{M}$. Precursor solutions for $\mathrm{Al}_{2} \mathrm{O}_{3}$ dielectrics were prepared by dissolving aluminum nitrate nonahydrate (98\%, Sigma-Aldrich) in 2-methoxyethanol (anhydrous, $99.8 \%$, Sigma-Aldrich) at a concentration of $0.5 \mathrm{M}$. Both solutions were stirred with a magnetic bar for $24 \mathrm{~h}$ at $65^{\circ} \mathrm{C}$. $\mathrm{MoS}_{2}$ dispersion was prepared by electrochemical exfoliation with molecular intercalants. First, we constructed a two-electrode electrochemical cell using $50 \mathrm{mg}$ of $\mathrm{MoS}_{2}$ crystal (HQ graphene) and graphite rod as cathode and anode, respectively. Tetraheptylammonium bromide (THAB, 99.0\%, Sigma-Aldrich) dissolved in acetonitrile (anhydrous, 99.8\%, Sigma-Aldrich) served as an electrolyte (concentration: $5 \mathrm{mg} \mathrm{mL}^{-1}$ ). To intercalate the $\mathrm{THA}^{+}$ions into the $\mathrm{MoS}_{2}$ crystal, a negative voltage of $7 \mathrm{~V}$ was applied to the cell for $1 \mathrm{~h}$. After the electrochemical reaction, as-intercalated crystal was rinsed with ethanol before sonication in polyvinylpyrrolidone (PVP, 40,000 $\mathrm{g} \mathrm{mol}^{-1}$, SigmaAldrich) solution in dimethylformamide (DMF) $\left(5 \mathrm{~mL} ; 22.2 \mathrm{mg} \mathrm{mL}^{-1}\right)$ for $30 \mathrm{~min}$. To remove un-exfoliated crystals, the dispersion was centrifuged at 4,000 rpm. The supernatant was subsequently centrifuged and washed twice with isopropanol (IPA) to remove residual PVP. After rinsing, the $\mathrm{MoS}_{2}$ flakes were redispersed in IPA at an optimized concentration. 
For SEA layer printing, the precursor solution was injected by a syringe pump at a rate of $2.5 \mathrm{~mL} \mathrm{~min}{ }^{-1}$ into the slot die head and out of the slot at the bottom. To slot-die coat the solution on desired substrates (p-doped 5-inch silicon wafer and ITO/glass), the stage of the slot die coater was transported at a constant rate of $10 \mathrm{~mm} \mathrm{~s}^{-1}$, followed by sintering for $2 \mathrm{~h}$ at $500{ }^{\circ} \mathrm{C}$ in a furnace. To prepare $\mathrm{Al}_{2} \mathrm{O}_{3}$ samples, the precursor solution was spin coated onto UV-treated p-doped Si wafers $(4,000 \mathrm{rpm}, 30 \mathrm{~s})$ followed by thermal annealing for $2 \mathrm{~h}$ at $500{ }^{\circ} \mathrm{C}$ in a furnace. ALD-deposited $\mathrm{Al}_{2} \mathrm{O}_{3}$ was prepared by standard atomic-layer deposition at a processing temperature of $100^{\circ} \mathrm{C}$.

$\mathrm{MoS}_{2}$ flake dispersion solutions were slot-die coated on top of dielectric layers with syringe pump injection rate of $2.5 \mathrm{~mL} \mathrm{~min}^{-1}$ and stage transport speed of $10 \mathrm{~mm} \mathrm{~s}^{-1}$. After $\mathrm{MoS}_{2}$ flake solutions were successfully coated, procedures to enhance the electrical properties of the semiconductor film were taken; a soft baking process of $60^{\circ} \mathrm{C}$ for $2 \mathrm{~min}$, chemical doping by $10 \mathrm{mg} \mathrm{mL}^{-1}$ of bis(trifluoromethane)sulfonimide (TFSI, Sigma-Aldrich) in 1,2-dichloroehtane (Sigma-Aldrich) solvent for $30 \mathrm{~min}$ and thermal annealing at $250^{\circ} \mathrm{C}$ for $30 \mathrm{~min}$. Both chemical doping by TFSI and thermal annealing were proceeded in an argon-filled glove box. To form channel arrays, $\mathrm{MoS}_{2}$ films were covered by a photoresist mask (AZ 5214E) patterned by conventional photolithography and then processed with reactive ion etching followed by photoresist lift-off. Source/drain electrodes were created by sequential thermal deposition of $\mathrm{Cr}(3 \mathrm{~nm})$ and $\mathrm{Au}(30 \mathrm{~nm})$ metal layers through a photolithographically patterned photoresist layer. Afterwards, a lift-off process was applied. To fabricate logic gates, an additional photolithography patterning of ITO local gate electrodes and sodium-embedded alumina were conducted before and after coating the sodium-embedded alumina.

\section{Characterization}

The surface topography and work function of the $\mathrm{MoS}_{2}$ thin-film were characterized using atomic force microscope and kelvin probe force microscope, respectively (Park Systems NX10). The elemental composition and chemical structure were investigated using $x$-ray photoelectron spectroscopy (ESCALAB $250 \mathrm{XI}$, Thermo Fisher Scientific) and Raman/photoluminescence spectroscopy (MAPLE-II). Electrical impedance of sodium-embedded alumina, solution-processed $\mathrm{Al}_{2} \mathrm{O}_{3}$ and $\mathrm{ALD}$-deposited $\mathrm{Al}_{2} \mathrm{O}_{3}$ was measured using VersaSTAT 4 potentiostat based on the metal-insulator-metal structure. Electrical properties for FETs and logic gates were characterized by using a vacuum probe station (under $10^{-4}$ Torr) and Keithley 4200 semiconductor electrometer.

\section{Declarations}

\section{Acknowledgements}

This research was supported by Creative Materials Discovery Program through the National Research Foundation of Korea (NRF) funded by Ministry of Science and ICT (NRF-2019M3D1A1078299) and 
National R\&D Program through the NRF funded by Ministry of Science and ICT (2021M3D1A2049315). J. Kang further acknowledges a National Research Foundation of Korea (NRF) grant funded by the Korean Government (MSIT) (2020R1C1C1009381).

\section{Author Contributions}

J.H.Cho and J.Kang initiated and supervised all the research. Yh.A.Kwon and Ji.K carried out and designed most of the experimental work and data analysis. D.G.Roe, D.Rhee and D.W.Kim assisted in the manuscript writing. S.B.Jo assisted in the data analysis. All authors discussed the results and contributed to the writing of the manuscript.

\section{Conflicts of interest}

There are no conflicts to declare.

\section{References}

1. Han, S.-J. et al. High-speed logic integrated circuits with solution-processed self-assembled carbon nanotubes. Nat. Nanotechnol. 12, 861-865 (2017).

2. Dai, X. et al. Solution-processed, high-performance light-emitting diodes based on quantum dots. Nature 515, 96-99 (2014).

3. Tsai, H. et al. Design principles for electronic charge transport in solution-processed vertically stacked 2D perovskite quantum wells. Nat. Commun. 9, 1-9 (2018).

4. McDonald, S. A. et al. Solution-processed PbS quantum dot infrared photodetectors and photovoltaics. Nat. Mater. 4, 138-142 (2005).

5. Wang, Y. X. et al. Field-effect transistors made from solution-grown two-dimensional tellurene. Nat. Electron. 1, 228-236 (2018).

6. Hanlon, D. et al. Liquid exfoliation of solvent-stabilized few-layer black phosphorus for applications beyond electronics. Nat. Commun. 6, 1-11 (2015).

7. Nicolosi, V., Chhowalla, M., Kanatzidis, M. G., Strano, M. S. \& Coleman, J. N. Liquid exfoliation of layered materials. Science $\mathbf{3 4 0}$ (2013).

8. Coleman, J. N. et al. Two-dimensional nanosheets produced by liquid exfoliation of layered materials. Science 331, 568-571 (2011). 
9. Carey, T. et al. Fully inkjet-printed two-dimensional material field-effect heterojunctions for wearable and textile electronics. Nat. Commun. 8, 1-11 (2017).

10. Kelly, A. G. et al. All-printed thin-film transistors from networks of liquid-exfoliated nanosheets. Science 356, 69-73 (2017).

11. Radisavljevic, B., Radenovic, A., Brivio, J., Giacometti, V. \& Kis, A. Single-layer MoS $_{2}$ transistors. Nat. Nanotechnol. 6, 147-150 (2011).

12. Lin, Z. Y., Huang, Y. \& Duan, X. F. Van der waals thin-film electronics. Nat. Electron. 2, 378-388 (2019).

13. Lin, Z. Y. et al. Solution-processable 2D semiconductors for high-performance large-area electronics. Nature 562, 254-258 (2018).

14. Lin, Z. et al. Solution processable colloidal nanoplates as building blocks for high-performance electronic thin films on flexible substrates. Nano Lett. 14, 6547-6553 (2014).

15. He, Q. et al. Fabrication of flexible $\mathrm{MoS}_{2}$ thin-film transistor arrays for practical gas-sensing applications. Small 8, 2994-2999 (2012).

16. Zheng, J. et al. High yield exfoliation of two-dimensional chalcogenides using sodium naphthalenide. Nat. Commun. 5, 1-7 (2014).

17. Gong, J. et al. Graphene quantum dots assisted exfoliation of atomically-thin 2D materials and asformed OD/2D van der waals heterojunction for HER. Carbon 184, 554-561 (2021).

18. Lee, J. et al. Monolayer optical memory cells based on artificial trap-mediated charge storage and release. Nat. Commun. 8, 1-8 (2017).

19. Di Bartolomeo, A. et al. Electrical transport and persistent photoconductivity in monolayer $\mathrm{MoS}_{2}$ phototransistors. Nanotechnology 28, 214002 (2017).

20. Lopez-Sanchez, O., Lembke, D., Kayci, M., Radenovic, A. \& Kis, A. Ultrasensitive photodetectors based on monolayer $\mathrm{MoS}_{2}$. Nat. Nanotechnol. 8, 497-501 (2013).

21. Fivaz, R. \& Mooser, E. Mobility of charge carriers in semiconducting layer structures. Phys. Rev. 163, 743 (1967).

22. Choi, M. et al. Flexible active-matrix organic light-emitting diode display enabled by $\mathrm{MoS}_{2}$ thin-film transistor. Sci. Adv. 4, eaas8721 (2018).

23. Chang, H. Y. et al. High-performance, highly bendable $\mathrm{MoS}_{2}$ transistors with high-k dielectrics for flexible low-power systems. ACS Nano 7, 5446-5452 (2013). 
24. Yang, J. et al. Improved growth behavior of atomic-layer-deposited high-k dielectrics on multilayer $\mathrm{MoS}_{2}$ by oxygen plasma pretreatment. ACS Appl. Mater. Interfaces 5, 4739-4744 (2013).

25. McDonnell, S. et al. $\mathrm{HfO}_{2}$ on $\mathrm{MoS}_{2}$ by atomic layer deposition: Adsorption mechanisms and thickness scalability. ACS Nano 7, 10354-10361 (2013).

26. Johnson, R. W., Hultqvist, A. \& Bent, S. F. A brief review of atomic layer deposition: From fundamentals to applications. Mater. Today 17, 236-246 (2014).

27. Oviroh, P. O., Akbarzadeh, R., Pan, D., Coetzee, R. A. M. \& Jen, T.-C. New development of atomic layer deposition: Processes, methods and applications. Sci. Technol. Adv. Mater. 20, 465-496 (2019).

28. Wei, J. et al. High-performance spin-coated aluminum oxide dielectric fabricated by a simple oxygen plasma-treatment process. J. Phys. D J PHYS D APPL PHYS 51, 365101 (2018).

29. Kim, B. J. et al. Water-gel for gating graphene transistors. Nano Lett. 14, 2610-2616 (2014).

30. Cho, J. H. et al. Printable ion-gel gate dielectrics for low-voltage polymer thin-film transistors on plastic. Nat. Mater. 7, 900-906 (2008).

31. Herlogsson, L. et al. Low-voltage polymer field-effect transistors gated via a proton conductor. Adv. Mater. 19, 97-101 (2007).

32. Larsson, O., Said, E., Berggren, M. \& Crispin, X. Insulator polarization mechanisms in polyelectrolytegated organic field-effect transistors. Adv. Funct. Mater. 19, 3334-3341 (2009).

33. Liu, Y., Guan, P., Zhang, B., Falk, M. L. \& Katz, H. E. Ion dependence of gate dielectric behavior of alkali metal ion-incorporated aluminas in oxide field-effect transistors. Chem. Mater. 25, 3788-3796 (2013).

34. Zhang, B., Liu, Y., Agarwal, S., Yeh, M.-L. \& Katz, H. E. Structure, sodium ion role, and practical issues for $\beta$-alumina as a high-k solution-processed gate layer for transparent and low-voltage electronics. ACS Appl. Mater. Interfaces 3, 4254-4261 (2011).

35. Liu, Y., McElhinny, K., Alley, O., Evans, P. G. \& Katz, H. E. Reduced-temperature solution-processed transparent oxide low-voltage-operable field-effect transistors. MRS Commun. 5, 605-611 (2015).

36. Pal, B. N., Dhar, B. M., See, K. C. \& Katz, H. E. Solution-deposited sodium beta-alumina gate dielectrics for low-voltage and transparent field-effect transistors. Nat. Mater. 8, 898-903 (2009).

37. Lin, P., Zhu, L. P., Li, D. \& Wang, Z. L. Defect repair for enhanced piezo-phototronic $\mathrm{MoS}_{2}$ flexible phototransistors. Journal of Materials Chemistry C 7, 14731-14738 (2019).

38. Namgung, S. D. et al. Influence of post-annealing on the off current of $\mathrm{MoS}_{2}$ field-effect transistors. Nanoscale Res. Lett. 10, 1-6 (2015). 
39. Van Keuls, F., Hu, X., Jiang, H. \& Dahm, A. Screening of the coulomb interaction in two-dimensional variable-range hopping. Phys. Rev. B 56, 1161 (1997).

40. Qiu, H. et al. Hopping transport through defect-induced localized states in molybdenum disulphide. Nat. Commun. 4 (2013).

41. Han, M. Y., Brant, J. C. \& Kim, P. Electron transport in disordered graphene nanoribbons. Phys. Rev. Lett. 104, 056801 (2010).

42. Upreti, T. et al. Experimentally validated hopping-transport model for energetically disordered organic semiconductors. Phys. Rev. Appl. 12, 064039 (2019).

43. He, T. et al. Crystal step edges can trap electrons on the surfaces of n-type organic semiconductors. Nat. Commun. 9, 1-9 (2018).

\section{Figures}

\section{Figure 1}

Device fabrication based on slot-die coating. (a) Schematic illustration of slot-die-coating of SEA and $\mathrm{MoS}_{2}$. (b) Optical image showing the slot-die coating setup for consecutive wafer-scale coating. (c) Image of slot-die-coated SEA and $\mathrm{MoS}_{2}$ on a 5-inch Si wafer. (d) Schematic illustration of $\mathrm{MoS}_{2} / \mathrm{SEA}$ transistor and atomic structure of $\mathrm{MoS}_{2}$ thin-film and SEA layer, respectively. (e) Transfer characteristics and $\mathrm{OM}$ image (inset) of $\mathrm{MoS}_{2}$ /SEA FET. (f) Color map of carrier mobility distribution. (g) Capacitance (left) and phase angle (right) properties of SEA with different frequencies. (h) Color map of effective capacitance distribution.

\section{Figure 2}

Device optimization and origin of high carrier mobility. (a) Schematic illustration of optimizing process of $\mathrm{MoS}_{2}$ semiconductor film. (b) Engineering of transfer characteristics by TFSI-treatment and thermal annealing on $\mathrm{MoS}_{2}$ transistor. (c) Conductivity $(\sigma)$ plotted against $\mathrm{T}^{-1 / 3}$ for sodium alumina gated devices measured at different gate voltages. 2D VRH fitted lines are shown for each gate voltage. (d) $T_{1}$ and (e) $T_{0}$ values extracted from 2D VRH model and Arrhenius plot, respectively. (f) KPFM image (top) representing spatial distribution of work function in sodium-embedded alumina film. 2D fast Fourier transform (bottom) of the KPFM image, which visualizes the oscillating amplitude in the $x$ and $y$ directions. (g) Gaussian fit of work function distribution for SEA and $\mathrm{Al}_{2} \mathrm{O}_{3}$. (h) Amplitude distribution of 
2D FFT image for SEA and $\mathrm{Al}_{2} \mathrm{O}_{3}$. (i) Schematic illustration of density of states (DOS) (left) and line profile of energy (right).

\section{Figure 3}

Applicability to large-area logic circuits. (a) Optical image of NOR, NOT, NAND, and SRAM logic gates. (b) Circuit diagram of NOT gate (inverter). (c) Voltage transfer characteristics (left) and resulting voltage gain (right) of NOT gate. (d) Circuit diagrams of NAND (top) and NOR (bottom) gates. (e) Input voltage signals (top) and the corresponding output voltage signals (bottom) of NAND and NOR gates. (f) Circuit diagram and $(\mathrm{g})$ input voltage signals and the corresponding output voltage signals of SRAM gate. The shaded regions indicate completely open input signals.

\section{Supplementary Files}

This is a list of supplementary files associated with this preprint. Click to download.

- SupplementaryinformationJHCHOJKANG.docx

- SupplementaryVideo1.mp4 